\title{
Influence of Planck foreground masks in the large angular scale quadrant CMB asymmetry
}

\author{
L. Santos ${ }^{1}$, P. Cabella ${ }^{2,3}$, T. Villela ${ }^{4}$, and W. Zhao ${ }^{1,5}$ \\ ${ }^{1}$ CAS Key Laboratory for Researches in Galaxies and Cosmology, University of Sciences and Technology of China, \\ Chinese Academy of Sciences, Hefei, 230026 Anhui, PR China \\ e-mail: larissa@ustc.edu.cn \\ 2 Università di Roma “Tor Vergata”, Dipartimento di Fisica, 00185 Rome, Italy \\ 3 INFN, Sezione di Roma Tor Vergata, 00173 Rome, Italy \\ 4 Instituto Nacional de Pesquisas Espaciais - INPE, Divisão de Astrofísica, 12227-010 São José dos Campos, SP, Brazil \\ 5 State Key Laboratory of Theoretical Physics, Institute of Theoretical Physics, Chinese Academy of Sciences, 100190 Beijing, \\ PR China
}

Received 10 June 2015 / Accepted 2 October 2015

\section{ABSTRACT}

Context. The measured cosmic microwave background (CMB) angular distribution shows high consistency with the $\Lambda$ CDM model, which predicts cosmological isotropy as one of its fundamental characteristics. However, isotropy violations were reported in CMB temperature maps of the Wilkinson Microwave Anisotropy Probe (WMAP) and confirmed by Planck satellite data.

Aims. Our purpose is to investigate the influence of different sky cuts (masks) employed in the analysis of CMB angular distribution, in particular in the excess of power in the southeastern quadrant (SEQ) and the lack of power in the northeastern quadrant (NEQ), found in both WMAP and Planck data.

Methods. We compared the two-point correlation function (TPCF) computed for each quadrant of the CMB foreground-cleaned temperature maps to 1000 Monte Carlo (MC) simulations generated assuming the $\Lambda$ CDM best-fit power spectrum using four different masks, from the least to the most severe one: mask-rulerminimal, UT78, U73, and U66. In addition to the quadrants and for a better understanding of these anomalies, we computed the TPCF using the mask-rulerminimal for circular regions in the map where the excess and lack of power are present. We also compared, for completeness, the effect of Galactic cuts (+/-10, 20, 25, and 30 degrees above/below the Galactic plane) in the TPCF calculations as compared to the MC simulations.

Results. We found consistent results for three masks, namely mask-rulerminimal, U73, and U66. The results indicate that the excess of power in the SEQ tends to vanish as the portion of the sky covered by the mask increases and the lack of power in the NEQ remains virtually unchanged. A different result arises for the newly released UT78 Planck mask. When this mask is applied, the NEQ is no longer anomalous. On the other hand, the excess of power in the SEQ becomes the most significant one among the masks. Nevertheless, the asymmetry between the SEQ and NEQ is independent of the mask and it disagrees with the isotropic model with at least $95 \%$ C.L.

Conclusions. We find that UT78 disagrees with the other analyzed masks, especially when considering the SEQ and the NEQ individual analysis. Most important, the use of UT78 washes out the anomaly in the NEQ. Furthermore, we find an excess of kurtosis, compared with simulations, in the NEQ for the regions not masked by UT78 but masked by the other masks, indicating that the previous result could be due to unremoved residual foregrounds by UT78.

Key words. cosmic background radiation - cosmology: observations - methods: data analysis - methods: statistical

\section{Introduction}

Cosmic microwave background $(\mathrm{CMB})$ radiation is one of the best cosmological observables, and it provides a powerful test of the so-called standard cosmological model, also known as the $\Lambda$ cold dark matter $(\Lambda \mathrm{CDM})$ model. The Planck satellite is the fourth-generation space mission devoted to CMB measurements, and it has recently released the most accurate CMB full-sky dataset to date. These results show an outstanding consistency with the spatially flat six-parameter standard model (Planck Collaboration XIII 2015).

Homogeneity and isotropy are fundamental properties of the $\Lambda \mathrm{CDM}$ cosmology, however deviations from statistical isotropy have been reported in CMB data throughout the years. One of these anomalies was first reported in the Cosmic Background Explorer (COBE) data (Smoot et al. 1992) and later confirmed by the Wilkinson Microwave Anisotropy Probe (WMAP) observations (Bennett et al. 2003; Hinshaw et al. 2007, 2009; Jarosik et al. 2011): a low quadrupole amplitude was detected, in disagreement with the predicted value from the standard model of cosmology. Other violations of isotropy were soon announced in WMAP data, including an alignment of the loworder multipoles (Bielewicz et al. 2004, 2005; Schwarz et al. 2004; Copi et al. 2004, 2006; de Oliveira-Costa et al. 2004; Land \& Magueijo 2005; Abramo et al. 2006; Gruppuso \& Burigana 2009; Frommert \& Ensslin 2010), the cold spot (Vielva et al. 2004; Cruz et al. 2005, 2007; Vielva 2010), the parity asymmetry (Kim \& Naselsky 2010; Gruppuso et al. 2011; Aluri \& Jain 2012; Hansen et al. 2012; Naselsky et al. 2012; Zhao 2014), and the north-south asymmetry (Eriksen et al. 2004a,b; Hansen et al. 2004a,b; Donoghue et al. 2005; Hoftuft et al. 2009; Paci et al. 2010; Pietrobon et al. 2010; Vielva et al. 2010). Closely related to the north-south asymmetry is the power asymmetry found between different quadrants of the CMB sky (Santos et al. 2012, 2014).

Most of the anomalies found in previous CMB observations were confirmed by Planck data, suggesting that they are not due to systematic effects in the instruments. Besides the 
confirmation from the Planck team (Planck Collaboration XXIII 2014; Planck Collaboration XVI 2015), Bernui et al. (2014) confirmed the north-south asymmetry in Planck data with a $98.1 \%$ C.L. and consideres it unlikely to be due to residual foregrounds. Akrami et al. (2014) also confirmed the hemispherical power asymmetry in both WMAP and Planck temperature sky maps. Other anomalies were also confirmed by different authors (see, for instance, Polastri et al. 2015 and Gurzadyan et al. 2014). However, the significance of CMB anomalies are far from consensus. On the other hand, results from Quartin et al. (2015) show that no significant power asymmetry is present in CMB full dataset when both Doppler and aberration effects are properly removed. Rassat et al. (2014) also claims that, after removing astrophysical and cosmological secondary effects, only the low quadrupole remains anomalous. The authors also conclude that masking the sky to avoid residual foregrounds has a bigger impact on CMB statistics than full-sky CMB analysis. It is essential to understand the origin of such features in CMB temperature distribution in order to either confirm the $\Lambda \mathrm{CDM}$ model or search for different explanations from the perspective of a new physics.

In this paper we examine how different sky cuts (from more conservative masks to less conservative ones) affect the excess of power found in the southeastern quadrant (SEQ) and the significant lack of power found in the northeastern quadrant (NEQ) of the sky. We use Planck 2015 foreground-cleaned temperature maps (for a detailed description, see Planck Collaboration IX 2015) and different masks as described in Sect. 2, in addition to the method we use to analyze the data. In Sects. 3 and 4, we discuss the results and present our conclusions.

\section{Method}

Our first step was to generate 1000 Monte Carlo (MC) simulated CMB maps with $N_{\text {side }}=256$ using the HEALPix (Hierarchical Equal Area and Isolatitude Pixelization) package Synfast (Gorski et al. 2005). These simulated CMB maps were created using the best-fit $\Lambda$ CMD model power spectrum from Planck (Planck Collaboration I 2014). As for CMB data, we consider the Planck 2015 release foreground-cleaned temperature sky maps, in particular the SMICA2 (the other maps are NILC2, SEVEM2, and Commander2). Owing to computational limitations and to our only being concerned with large angular scales, all maps were degraded to $N_{\text {side }}=64$ (including the MC maps). We then divided each simulated map as well as each CMB map into four quadrants: SEQ, NEQ, southwestern (SWQ) and northwestern (NWQ). Furthermore, instead of dividing the sky into quadrants, we also chose circular regions (in the CMB map as well as in the simulations) where we found the excess and lack of power in the CMB sky.

At this stage, we mask pixels that can still be contaminated by residual foregrounds in every CMB map. Since we are comparing the real data with simulations, the same procedure must be done to the MC maps. Four different masks with different sky cuts are considered. The masks provided by the Planck team are three: from the first data release, mask-rulerminimal, and U73 (they cut $16.35 \%$ and $25.17 \%$ of the sky for $N_{\text {side }}=64$, respectively); and from the second data release, mask UT78 (cuts $21.33 \%$ of the sky for $N_{\text {side }}=64$ ). The fourth mask is named U66 and is the most severe one (cuts $32.59 \%$ of the sky for $\left.N_{\text {side }}=64\right)$. It was constructed by Axelsson et al. (2015) and it is publicly available.

In the case where we considered circular regions, we only used only the least severe mask (mask-rulerminimal) because of computational limitations. We also tested different radii for each chosen region. We restricted ourselves to radius that run from $60^{\circ}$ to $80^{\circ}$ for two reasons: we are considering large angular scale asymmetries and for a direct comparison with the previously chosen quadrants (in the number of pixels used in the analysis).

The third step is to calculate the two-point correlation function (TPCF) for each quadrant or circular region in every map (CMB data and simulations), applying each of the described masks at a time (in the case where the sky into divided in quadrants). We define the TPCF as the average product between the temperature of all pairs of pixels separated by an angular distance $\gamma$ in each analyzed masked sky map:

$c(\gamma) \equiv\left\langle T\left(\boldsymbol{n}_{\mathrm{p}}\right) T\left(\boldsymbol{n}_{\mathrm{q}}\right)\right\rangle$.

The temperature fluctuations of the pixels $\mathrm{p}$ and $\mathrm{q}$ are described by $T\left(\boldsymbol{n}_{\mathrm{p}}\right)$ and $T\left(\boldsymbol{n}_{\mathrm{q}}\right)$, respectively. Moreover, these pixels are defined by the coordinates $\left(\theta_{\mathrm{p}}, \phi_{\mathrm{p}}\right)$ and $\left(\theta_{\mathrm{q}}, \phi_{\mathrm{q}}\right)$, where $0^{\circ} \leq \phi \leq$ $360^{\circ}$ and $-90^{\circ} \leq \theta \leq 90^{\circ}$. The angular distance between two generic pixels is given by

$\cos \gamma=\cos \theta_{\mathrm{p}} \cos \theta_{\mathrm{q}}+\sin \theta_{\mathrm{p}} \sin \theta_{\mathrm{q}} \cos \left(\phi_{\mathrm{p}}-\phi_{\mathrm{q}}\right)$.

The next step is to quantify the results by calculating an rms-like quantity, $\sigma$, defined in Bernui et al. (2006) for each TPCF curve:

$\sigma=\sqrt{\frac{1}{N_{\text {bins }}} \sum_{i=1}^{N_{\text {bins }}} f_{i}^{2}}$,

where $f_{i}$ corresponds to the TPCF for each bin $i$ and $N_{\text {bins }}=90$.

Finally, we compare both the TPCF curves and the rms-like quantity $\sigma$ for Planck $\mathrm{CMB}$ maps and for the simulated $\Lambda \mathrm{CDM}$ model MC maps in each quadrant using four different sky masks. We discuss the results in the next section.

\section{Results and discussion}

The TPCF computed for each quadrant of SMICA2 map is shown in Figs. 1-4, using masks mask-rulerminimal, U73, UT78, and U66, respectively. Using the quantity $\sigma$, defined in Eq. (3), we quantify the results and compare them with the MC simulated maps for each mask described above. Moreover, we can see that the TPCF computed for the maps SMICA2, NILC2, SEVEM2, and Commander2 using mask UT78 agree, as seen in Fig. 5.

The analysis shows consistent results for three masks: maskrulerminimal, U73, and U66. For all these cases, we found a lack of large-angle temperature correlation in the NEQ. The probability that the exactly same quadrant in the simulations presents the lack of correlation observed in SMICA2 is $0.2 \%$ for both maskrulerminimal and U73, and $1.4 \%$ for U66. If we allow that at least one quadrant, among all four of them, in each simulated map shares the absence of correlation presented in the data, we find values up to $5.4 \%$ for U66. The excess of power in the SEQ also follows a pattern for these three masks: it tends to vanish as the number of excluded pixels gets larger, i.e., when the masks are more severe. The probability that the excess of power occurs in the simulated maps varies, depending on which mask is being taken into account, from $8.2 \%$ to $28.9 \%$, if we only consider the SEQ of the simulations. On the other hand, if we establish that at least one quadrant among all in every simulation can share the excess of power found in the data, the probability increases from $27.1 \%$ to $64.1 \%$. For details see Tables 1 and 5 . 
L. Santos et al.: Influence of Planck foreground masks in the large angular scale quadrant CMB asymmetry

Table 1. Probabilities of finding higher or lower $\sigma$ values in exactly the same quadrant of the simulated MC maps than in Planck SMICA2 map.

\begin{tabular}{lcrrrrrrr}
\hline \hline Map & $\sigma_{\mathrm{SEQ}}$ & $\mathrm{P}^{a}$ & $\sigma_{\mathrm{SWQ}}$ & $\mathrm{P}^{b}$ & $\sigma_{\mathrm{NEQ}}$ & $\mathrm{P}^{c}$ & $\sigma_{\mathrm{NWQ}}$ & P4 $^{d}$ \\
\hline SMICA2 + mask-rulerminimal & 1891.99 & $8.2 \%$ & 506.74 & $10.8 \%$ & 308.32 & $0.2 \%$ & 420.39 & $2.4 \%$ \\
SMICA2 + U73 & 1356.25 & $20.3 \%$ & 567.98 & $16.2 \%$ & 302.18 & $0.2 \%$ & 810.99 & $54.3 \%$ \\
SMICA2 + UT78 & 2373.69 & $2.3 \%$ & 985.71 & $46.4 \%$ & 677.26 & $29.1 \%$ & 558.94 & $12.9 \%$ \\
SMICA2 + U66 & 1257.18 & $28.9 \%$ & 829.16 & $45.2 \%$ & 374.49 & $1.4 \%$ & 1233.65 & $24.3 \%$ \\
\hline
\end{tabular}

Notes. We show the effect in each quadrant of different masks provided by the Planck team. ${ }^{(a)}$ Probability of finding $\sigma_{\mathrm{MC}}>\sigma_{\mathrm{SEQ}}$; ${ }^{(b)}$ probability of finding $\sigma_{\mathrm{MC}}<\sigma_{\mathrm{SWQ}}{ }^{(c)}$ probability of finding $\sigma_{\mathrm{MC}}<\sigma_{\mathrm{NEQ}}{ }^{\left({ }^{(d)}\right)}$ probability of finding $\sigma_{\mathrm{MC}}<\sigma_{\mathrm{NWQ}}$. An exception was made for SMICA2 + U66 where the probability of finding $\sigma_{\mathrm{MC}}>\sigma_{\mathrm{NWQ}}$ was calculated instead.
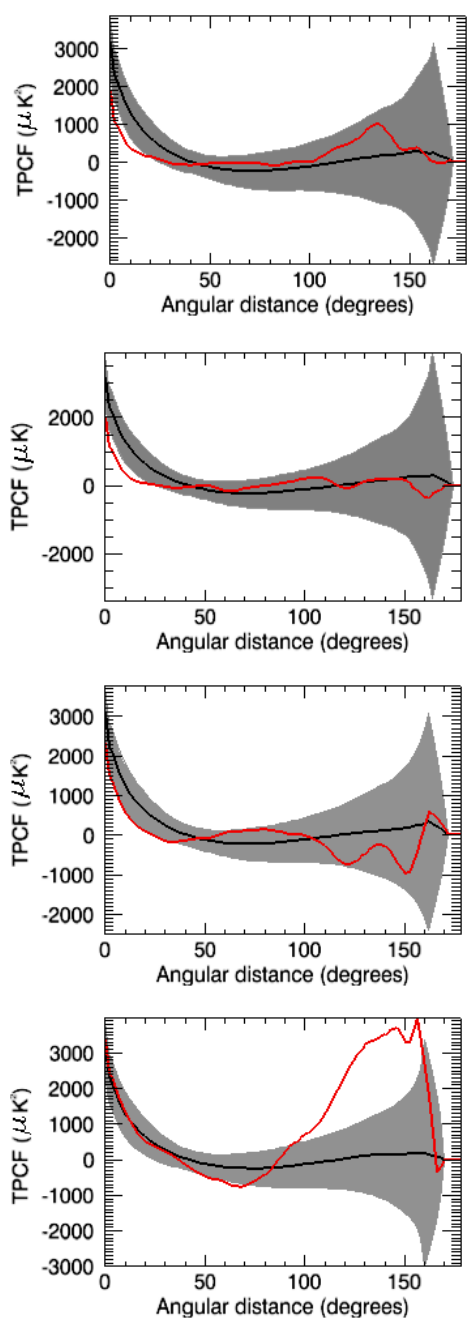

Fig. 1. TPCF curves computed for the main Planck second data release temperature foreground-cleaned map (SMICA2) (red curve) using mask-rulerminimal. We smoothed the curves using the smooth function from Interactive Data Language (IDL) for illustration purposes only (in the calculations we use the original calculated values for the TPCF). From top to bottom, NWQ, NEQ, SWQ, and SEQ appear as solid red lines. The shadow part depicts the standard deviation intervals (68\% C.L) for 1000 simulated maps produced with the $\Lambda$ CDM spectrum. The black curve is the mean TPCF considering the MC simulated maps.

Briefly, we can say that even though there is an excess of power in the SEQ, it does agree with the $\Lambda$ CDM model when we apply mask-rulerminimal, U73, and U66 masks to SMICA2. However, we quantified the probability that both features, namely the lack of correlation in the NEQ and the excess of power in the SEQ, occur at the same time in CMB data as the
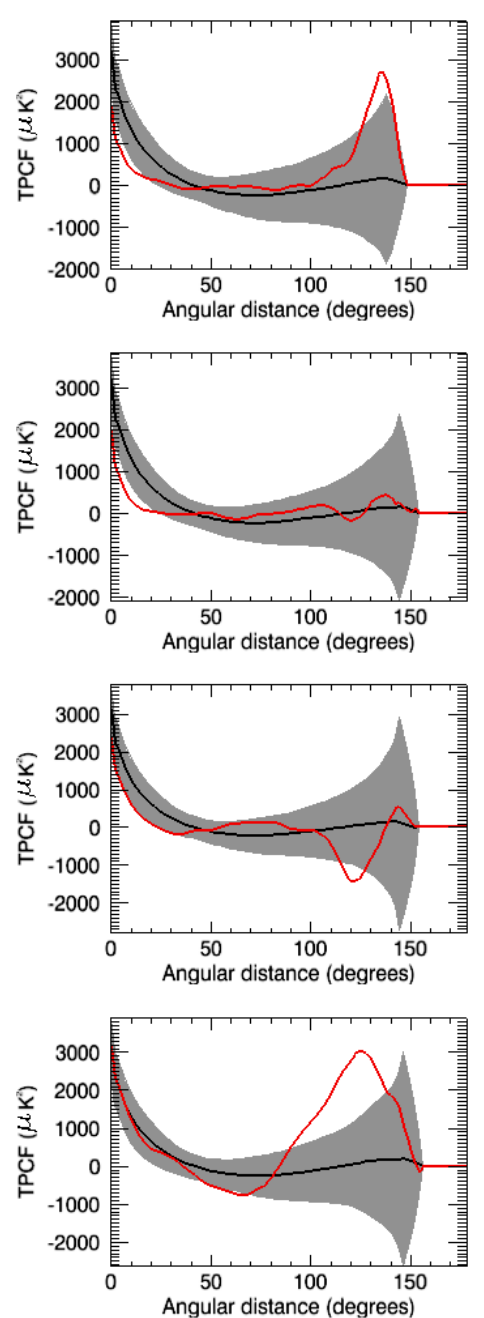

Fig. 2. TPCF curves computed for SMICA2 (red curve) using U73.

asymmetry between both quadrants. We find that $<0.1 \%, 1.4 \%$, and $4.6 \%$ of the simulated maps have the same asymmetry as the SMICA2 CMB map for mask-rulerminimal, U73 and U66, respectively (see Table 3). Once more, if we allow that the asymmetry can happen between any pair of quadrants of a simulated map, the above probabilities increase to $1.4 \%, 6.3 \%$, and $20.4 \%$, no longer anomalous for conservative masks. Table 4 compares the values of $\sigma_{\mathrm{SEQ}} / \sigma_{\mathrm{NEQ}}$ found in the data with the mean values found in the simulations for $68 \%$ C.L., 95\% C.L and 99.7\% C.L.

A different result was found for the new UT78 2015 Planck mask. In contrast to all other masks, when UT78 was applied to the data, we found that the NEQ is no longer anomalous, meaning that now there is a large-angle temperature correlation in this 
Table 2. Probabilities of finding higher or lower $\sigma$ values in at least one quadrant of the simulated MC maps than in the Planck SMICA2 map.

\begin{tabular}{lcccccrrr}
\hline \hline Map & $\sigma_{\mathrm{SEQ}}$ & $\mathrm{P}^{a}$ & $\sigma_{\mathrm{SWQ}}$ & $\mathrm{P}^{b}$ & $\sigma_{\mathrm{NEQ}}$ & $\mathrm{P}^{c}$ & $\sigma_{\mathrm{NWQ}}$ & $\mathrm{P}^{d}$ \\
\hline SMICA2 + mask-rulerminimal & 1891.99 & $27.1 \%$ & 506.74 & $25.3 \%$ & 308.32 & $0.6 \%$ & 420.39 & $8.8 \%$ \\
SMICA2 + U73 & 1356.25 & $43.8 \%$ & 567.98 & $55.1 \%$ & 302.18 & $1 \%$ & 810.99 & $88.6 \%$ \\
SMICA2 + UT78 & 2373.69 & $10.9 \%$ & 985.71 & $94.6 \%$ & 677.26 & $64.1 \%$ & 558.94 & $41.1 \%$ \\
SMICA2 + U66 & 1257.18 & $64.1 \%$ & 829.16 & $85.9 \%$ & 374.49 & $5.4 \%$ & 1233.65 & $65.6 \%$ \\
\hline
\end{tabular}

Notes. We show the effect in each quadrant of different masks provided by the Planck team. ${ }^{(a)}$ Probability of finding $\sigma_{\mathrm{MC}}>\sigma_{\mathrm{SEQ}}$; ${ }^{(b)}$ probability of finding $\sigma_{\mathrm{MC}}<\sigma_{\mathrm{SWQ}} ;{ }^{(c)}$ probability of finding $\sigma_{\mathrm{MC}}<\sigma_{\mathrm{NEQ}}$; ${ }^{(d)}$ probability of finding $\sigma_{\mathrm{MC}}<\sigma_{\mathrm{NWQ}}$. An exception was made for SMICA2 + U66 where the probability of finding $\sigma_{\mathrm{MC}}>\sigma_{\mathrm{NWQ}}$ was calculated instead.
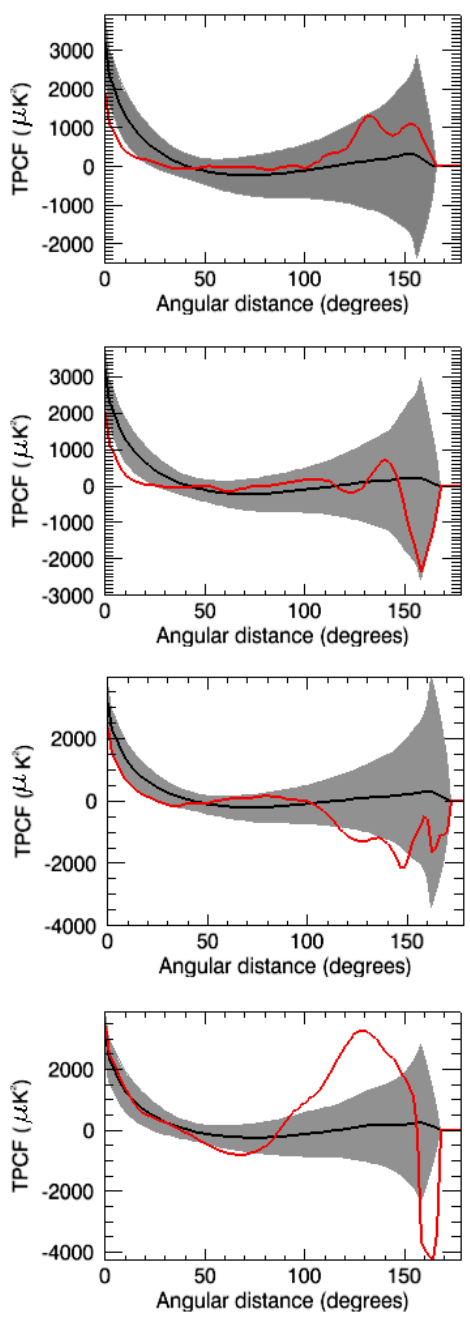

Fig. 3. TPCF curves computed for SMICA2 (red curve) using UT78.

quadrant, which brings it to agreement with the behavior defined by the standard cosmological model. On the other hand, only $2.3 \%$ of the simulations have the excess of power in the SEQ found in SMICA2. In other words, the excess of power in the SEQ is the greatest when we use UT78. If any quadrant in the simulation can account for this excess of power, the probability increases to $10.9 \%$ (see again Tables 1 and 5). The chance that the asymmetry between NEQ and SEQ occurs in the simulations for the exact same pair of quadrants is of $2.7 \%$ and $17.1 \%$ between any pair of quadrants in each simulated map.

For every mask used, therefore, we still find an anomalous asymmetry between the NEQ and the SEQ, taking the same pair of quadrants in the simulations into account (considering
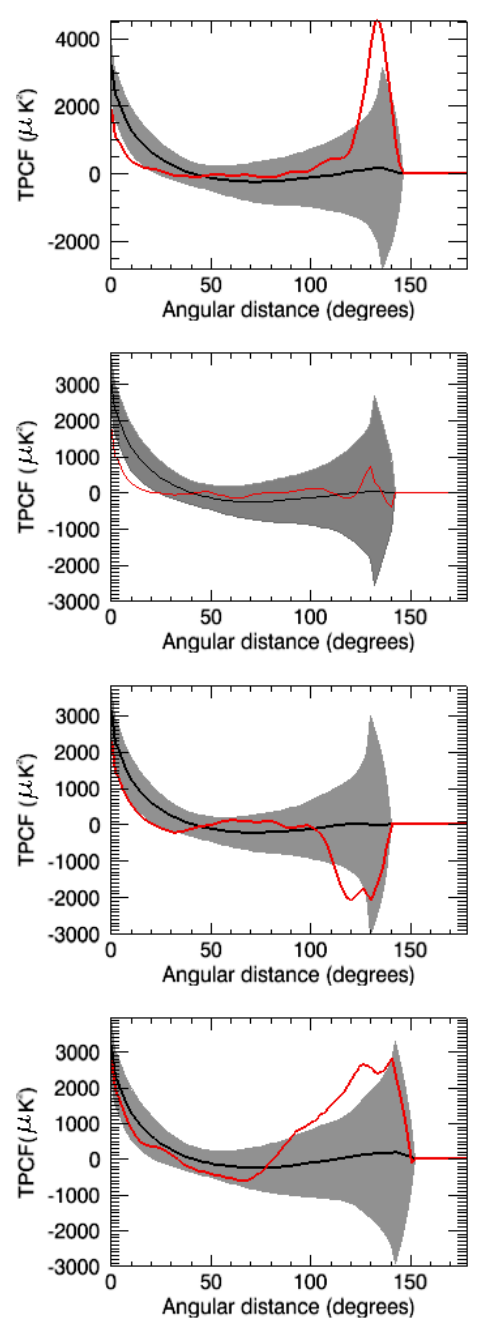

Fig. 4. TPCF curves computed for SMICA2 (red curve) using U66.

that in this case they have the same number of pixels as in the CMB map). However, the reason we find the asymmetry in UT78 is different from the reason we find it in mask-rulerminimal, U73 and UT78. To investigate this problem, we combined UT78 with each one of the other masks and calculated the TPCF and its correspondent $\sigma$ value for each quadrant of the SMICA2 map. The results can be seen in Table 5. It is possible to notice that the new results agree with the previous ones presented in this paper for only mask-rulerminimal, U73, and U66. The lack of correlation in the NEQ is still present in the data for UT78 combined with any of the other masks. The excess of power in the SEQ also follows the pattern described previously: it decreases as the mask becomes more severe. 

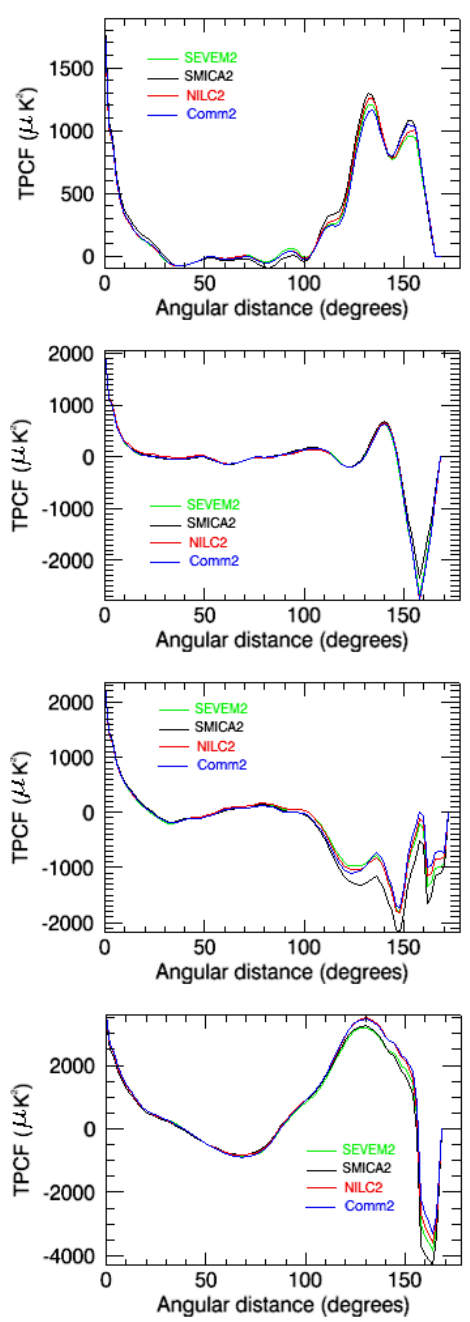

Fig. 5. Comparison between the Planck foreground-cleaned maps using the UT78 mask (SMICA2, NILC2, SEVEM2, and commander2). From top to bottom, the curves refer to the NWQ, NEQ, SWQ, and SEQ.

Table 3. Calculated probabilities of finding the asymmetry between the SEQ and the NEQ equal to or higher than those found in Planck data in the MC simulations using the Planck masks described previously considering the $\Lambda \mathrm{CDM}$ model.

\begin{tabular}{lcrrr}
\hline \hline Map & $\sigma_{\mathrm{SEQ}} / \sigma_{\mathrm{NEQ}}$ & $\mathrm{P}^{a}$ & $\mathrm{P}^{b}$ & $\mathrm{P}^{c}$ \\
\hline SMICA2 + mask-rulerminimal & 6.1 & $<0.1 \%$ & $0.3 \%$ & $1.4 \%$ \\
SMICA2 + U73 & 4.5 & $1.4 \%$ & $2.8 \%$ & $6.3 \%$ \\
SMICA2 + UT78 & 3.5 & $2.7 \%$ & $4.2 \%$ & $17.1 \%$ \\
SMICA2 + U66 & 3.4 & $4.6 \%$ & $9.7 \%$ & $20.4 \%$ \\
\hline
\end{tabular}

Notes. ${ }^{(a)}$ Probability of finding the asymmetries in the simulations for exactly same configuration as in the SMICA map. ${ }^{(b)}$ Probability of finding the asymmetries between the SEQ quadrant and any other quadrant in the simulations. ${ }^{(c)}$ Probability of finding the asymmetries between any pair of quadrants in the simulations.

Furthermore, for completeness, we calculated the TPCF and the correspondending $\sigma$ value for the SMICA2 map by performing simple symmetric Galactic cuts of $10^{\circ}, 20^{\circ}, 25^{\circ}$, and $30^{\circ}$. We again found that the excess of power in the SEQ decreases as the Galactic cut gets more severe. Moreover, for a more severe symmetric cut of $30^{\circ}$, the result agrees with the ones for maskrulerminimal, U73 and U66 with a significant lack of power in
Table 4. Mean, $68.2 \%, 95 \%$, and $99.7 \%$ C.L. values of $\sigma_{\mathrm{SEQ}} / \sigma_{\mathrm{NEQ}}$ using the simulated $\mathrm{CMB}$ maps considering the $\Lambda \mathrm{CDM}$ model for the four studied masks.

\begin{tabular}{lcccc}
\hline \hline Masks & Mean & $68 \%$ C.L. & 95\% C.L. & $99.7 \%$ C.L. \\
\hline mask-rulerminimal & 1.14 & 1.13 & 2.55 & 4.45 \\
U73 & 1.33 & 1.22 & 2.85 & 6.31 \\
UT78 & 1.21 & 1.13 & 2.82 & 5.26 \\
U66 & 1.37 & 1.38 & 3.16 & 6.32 \\
\hline
\end{tabular}

Table 5. $\sigma$ values for each quadrant in SMICA2 temperature map using combined Planck masks.

\begin{tabular}{lcccc}
\hline \hline Combined masks & $\sigma_{\mathrm{SEQ}}$ & $\sigma_{\mathrm{SWQ}}$ & $\sigma_{\mathrm{NEQ}}$ & $\sigma_{\mathrm{NWQ}}$ \\
\hline mask-rulerminimal + UT78 & 1585.87 & 839.41 & 378.95 & 536.62 \\
U73 + UT78 & 1404.45 & 556.13 & 315.54 & 795.92 \\
U66+ UT78 & 1336.02 & 1019.29 & 379.08 & 1221.22 \\
\hline
\end{tabular}

Table 6. $\sigma$ values for each quadrant in SMICA2 temperature map using symmetric Galactic cuts.

\begin{tabular}{lcccc}
\hline \hline Symmetric Galactic cut & $\sigma_{\mathrm{SEQ}}$ & $\sigma_{\mathrm{SWQ}}$ & $\sigma_{\mathrm{NEQ}}$ & $\sigma_{\mathrm{NWQ}}$ \\
\hline$+/-10^{\circ}$ & 2088.76 & 529.51 & 569.48 & 464.12 \\
$+/-20^{\circ}$ & 1250.25 & 635.21 & 520.94 & 410.29 \\
$+/-25^{\circ}$ & 730.22 & 921.12 & 518.85 & 333.44 \\
$+/-30^{\circ}$ & 500.31 & 540.26 & 289.69 & 380.30 \\
\hline
\end{tabular}

the NEQ (see Table 6 for details). To these symmetric Galactic cuts we also added the point source masks provided by the Planck team in the first and second data releases, obtaining no significant change in the results as presented in Table 6.

Finally, to check that the results obtained for UT78 could be due to unremoved foregrounds, we calculated histograms and their statistics (skewness and kurtosis) for the regions in the sky not covered by UT78 but covered by mask-rulerminimal or U73 or U66. We compared the values obtained for both quantities using SMICA2 and the simulations. We especially found interesting results for the NEQ: the value for the kurtosis in these regions is always above three for the data and on average not bigger than 2.42 for the simulations as can be seen in Table 7 . The value of the kurtosis for the regions uncovered by UT78, but covered by U73 in the NEQ (called UT78-U73), is 4.94, which is higher when compared with the average value of 2.21 for the simulations. We did not find any simulated map among 1000 simulations with such a high value of kurtosis in the same region of the sky. This result means that CMB temperature distribution in the analyzed regions is highly concentrated around the mean if compared to the $\Lambda \mathrm{CDM}$ simulations.

In addition to the previous analysis, we calculated the TPCF where we found the excess and lack of power in CMB sky using circular regions and compared with the results for the simulated sky maps. We found that the biggest excess of power falls in the region centered at $(\phi, \theta)=\left(270^{\circ}, 135^{\circ}\right)($ from now on Region 1$)$ (HEALPix convention) and radius, $r=80^{\circ}$ (11.488 pixels available). The results can be seen in Fig. 6, where we also compare the result for Region 1 for $r=80^{\circ}, r=77^{\circ}$, and $r=83^{\circ}$. The excess of power tends to decrease for radius different than $r=80^{\circ}$ (check Table 8 for the corresponding $\sigma$ values for the TPCF in Region 1 using $r=80^{\circ}, r=77^{\circ}$, and $r=83^{\circ}$ ). 
Table 7. Comparison of the histograms statistics (skewness and kurtosis) in the regions where UT78 is not masked and each of the other masks are masked using SMICA2 and the mean values for the MC simulations.

\begin{tabular}{|c|c|c|c|c|c|c|c|c|}
\hline Mask & SEQ $_{\text {SMICA2 }}$ & $\mathrm{SEQ}_{\mathrm{MC}}$ & $\mathrm{SWQ}_{\mathrm{SMICA} 2}$ & $\mathrm{SWQ}_{\mathrm{MC}}$ & $\mathrm{NEQ}_{\text {SMICA2 }}$ & $\mathrm{NEQ}_{\mathrm{MC}}$ & $\mathrm{NWQ}_{\text {SMICA2 }}$ & $\mathrm{NWQ}_{\mathrm{MC}}$ \\
\hline UT78- mask-rulerminimal & $(1.53,2.63)$ & $(1.47,2.15)$ & $(1.23,1.24)$ & $(1.38,1.89)$ & $(1.58,3.14)$ & $(1.41,1.94)$ & $(1.64,3.99)$ & $(1.36,1.78)$ \\
\hline UT78-U73 & $(1.76,3.83)$ & $(1.52,2.28)$ & $(1.58,2.89)$ & $(1.50,2.22)$ & $(1.94,4.94)$ & $(1.49,2.21)$ & $(1.58,2.77)$ & $(1.50,2.21)$ \\
\hline UT78-U66 & $(1.54,2.46)$ & $(1.57,2.46)$ & $(1.47,1.92)$ & $(1.56,2.42)$ & $(1.76,3.57)$ & $(1.56,2.42)$ & $(1.55,2.50)$ & $(1.54,2.37)$ \\
\hline
\end{tabular}

Table 8. $\sigma$ values for each circular region in SMICA2 temperature map considering different radius.

\begin{tabular}{lcccccc}
\hline \hline Region & Radius & $\sigma$ & Radius & $\sigma$ & Radius & $\sigma$ \\
\hline 1 & $77^{\circ}$ & 1474.08 & $80^{\circ}$ & 1923.46 & $83^{\circ}$ & 1485.98 \\
2 & $60^{\circ}$ & 328.22 & $70^{\circ}$ & 366.67 & $80^{\circ}$ & 386.83 \\
3 & $60^{\circ}$ & 414.50 & $70^{\circ}$ & 384.00 & $80^{\circ}$ & 524.98 \\
\hline
\end{tabular}
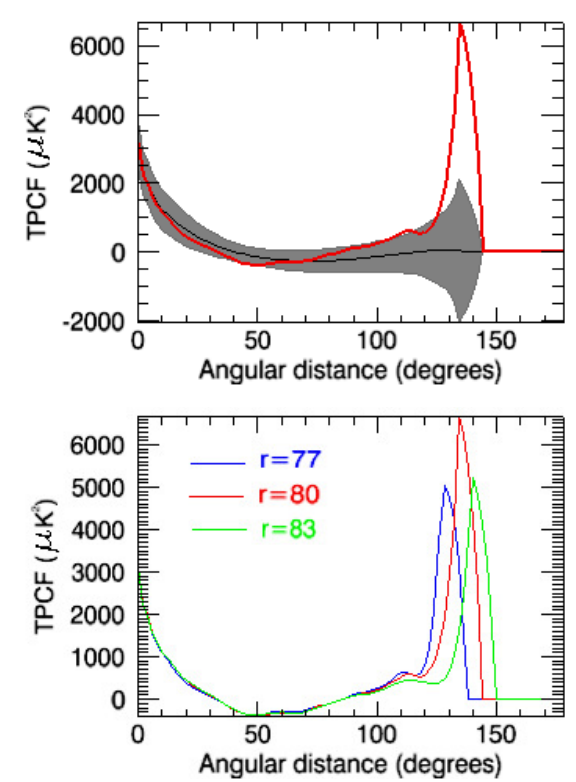

Fig. 6. Top: TPCF curve computed for SMICA2 (red curve) using maskrulerminimal in a circular region centered at $(\phi, \theta)=\left(270^{\circ}, 135^{\circ}\right)$ and radius, $r=80^{\circ}$. The shadow part depicts the standard deviation intervals (68\% C.L) for 1000 simulated maps produced with the $\Lambda$ CDM spectrum. The black curve is the mean TPCF considering the MC simulated maps. Bottom: TPCF computed for SMICA2 using mask-rulerminimal in a circular region centered at $(\phi, \theta)=\left(270^{\circ}, 135^{\circ}\right)$ and radius, $r=$ $77^{\circ}, 80^{\circ}$, and $83^{\circ}$

In the same way, we find a significant lack of power for regions centered at $(\phi, \theta)=\left(270^{\circ}, 45^{\circ}\right)$ (Region 2) and $(\phi, \theta)=$ $\left(225^{\circ}, 45^{\circ}\right)$ (Region 3 ) for radius, $r=60$ (6988 pixels), and $r=70$ (9307 pixels), respectively (see Figs. 7 and 8 for comparison of the TPCF in SMICA2 and simulations). If we consider Region 2 , we find that if we vary the radius from $60^{\circ}$ to $80^{\circ}$, the lack of power in this region remains nearly unchanged (see Table 8).

We find that both the excess of power in Region $1\left(80^{\circ}\right)$ and lack of power in Regions $2\left(60^{\circ}\right)$ and $3\left(70^{\circ}\right)$ have a low probability of occuring in the simulations which are $1 \%, 2.1 \%$, and $5.3 \%$, respectively. These later results agree with the previous ones (when we divided the sky in quadrants) as expected. We can compare the results for the quadrants and for the new circular regions by looking at Table 1 (first line, for mask-rulerminimal) and Table 9.
Table 9. Probabilities of finding higher or lower $\sigma$ values in exactly the same circular region of the simulated MC maps than in Planck SMICA2 map using mask-rulerminimal.

\begin{tabular}{lccr}
\hline \hline Region & Radius & $\sigma$ & Probability \\
\hline 1 & $80^{\circ}$ & 1923.50 & $1.0 \%^{a}$ \\
2 & $60^{\circ}$ & 328.22 & $2.1 \%^{b}$ \\
3 & $70^{\circ}$ & 384.00 & $5.3 \%^{b}$ \\
\hline
\end{tabular}

Notes. ${ }^{(a)}$ Probability of finding $\sigma_{\mathrm{MC}}>\sigma_{1}$; ${ }^{(b)}$ probability of finding $\sigma_{\mathrm{MC}}<\sigma_{2,3}$.
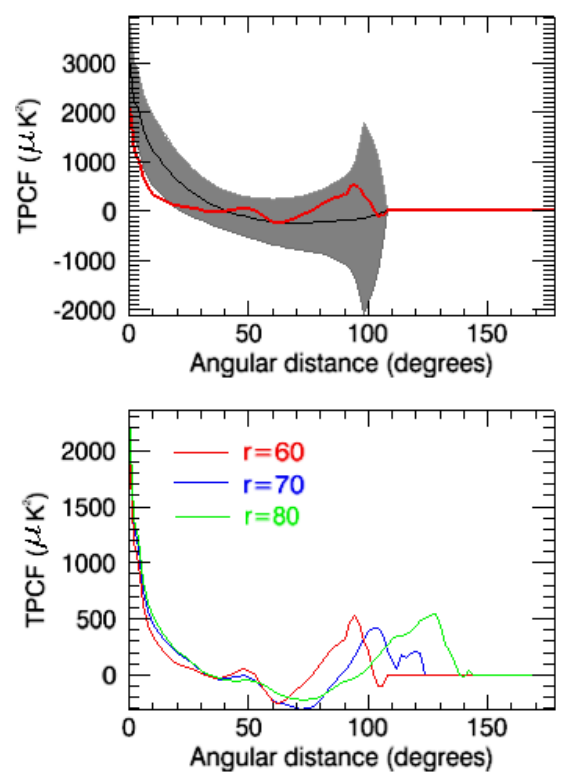

Fig. 7. Top: TPCF curve computed for SMICA2 (red curve) using maskrulerminimal in a circular region centered at $(\phi, \theta)=\left(270^{\circ}, 45^{\circ}\right)$ and radius, $r=60^{\circ}$. The shadow part depicts the standard deviation intervals (68\% C.L) for 1000 simulated maps produced with the $\Lambda$ CDM spectrum. The black curve is the mean TPCF considering the MC simulated maps. Bottom: TPCF computed for SMICA2 using mask-rulerminimal in a circular region centered at $(\phi, \theta)=\left(270^{\circ}, 45^{\circ}\right)$ and radius, $r=$ $60^{\circ}, 70^{\circ}$, and $80^{\circ}$.

\section{Conclusion}

We found good agreement among the results when considering mask-rulerminimal, U73, and U66 in the analysis of CMB angular distribution. For all these masks, we confirmed previous results in which CMB data present a significant lack of large-angle temperature correlation in the NEQ, which is anomalous if we assume the $\Lambda \mathrm{CDM}$. In the same way, we found an excess of power in SEQ that decreases as the mask becomes more severe. Nevertheless, the asymmetry between the SEQ and the NEQ is confirmed in CMB data for mask-rulerminimal, U73, and U66 with at least 95\% C.L. The excess of power in the SEQ computed when applying a symmetric cut around the 

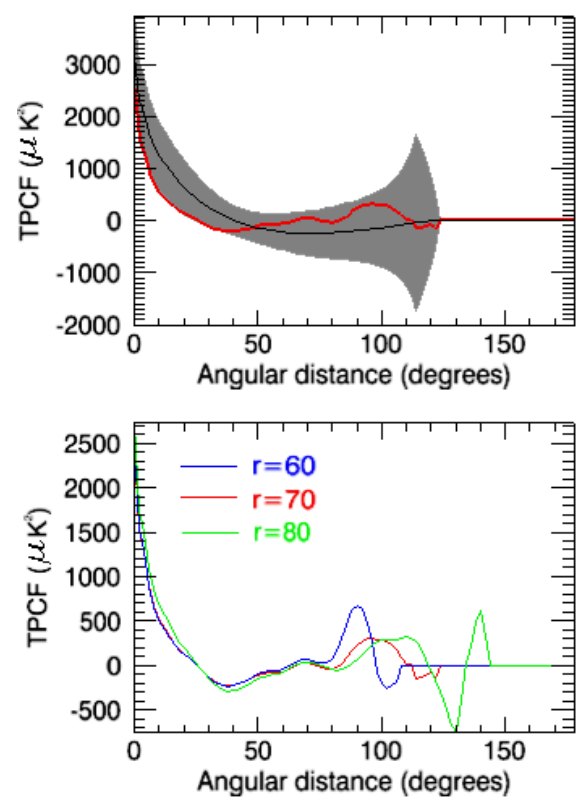

Fig. 8. Top: TPCF curve computed for SMICA2 (red curve) using maskrulerminimal in a circular region centered at $(\phi, \theta)=\left(225^{\circ}, 45^{\circ}\right)$ and radius, $r=70^{\circ}$. The shadow part depicts the standard deviation intervals (68\% C.L) for 1000 simulated maps produced with the $\Lambda$ CDM spectrum. The black curve is the mean TPCF considering the MC simulated maps. Bottom: TPCF computed for SMICA2 using mask-rulerminimal in a circular region centered at $(\phi, \theta)=\left(225^{\circ}, 45^{\circ}\right)$ and radius, $r=$ $60^{\circ}, 70^{\circ}$ and $80^{\circ}$.

Galactic plane is also in agreement with mask-rulerminimal, U73, and U66. The lack of power in the NEQ also agrees with the cited masks for a conservative symmetric cut of $30^{\circ}$.

A different result appears in the analysis for the new Planck mask released by the Planck team in 2015. In this case, even though the asymmetry between the SEQ and NEQ is still confirmed in the data, the NEQ agrees with the $\Lambda$ CMD model, and the SEQ presents the biggest excess of power among all the other tested masks. Further calculations show that regions in the sky (especially in the NEQ) which are uncovered by UT78 but covered by other masks, have a high value of kurtosis in the data, which disagrees with the values found in the simulations. This result suggests that UT78 leaves residual foregrounds in the data unmasked, and that if so, they are unsuitable for CMB cosmological analysis.

Finally, as expected, by choosing different regions in the CMB sky, we conclude that the power asymmetry does not depend on our previous choice of quadrants.

Acknowledgements. The authors acknowledge the use of HEALPix packages, of the Legacy Archive for Microwave Background Data Analysis (LAMBDA), and of the Planck Legacy Archive (PLA). The development of Planck has been supported by ESA, CNES, and CNRS/INSU-IN2P3-INP (France); ASI, CNR, and INAF (Italy); NASA and DoE (USA); STFC and UKSA (UK); CSIC, MICINN, and JA (Spain); Tekes, AoF, and CSC (Finland); DLR and MPG (Germany); CSA (Canada); DTU Space (Denmark); SER/SSO (Switzerland); RCN (Norway); SFI (Ireland); FCT/MCTES (Portugal); and the development of Planck has been supported by ESA, CNES, and CNRS/INSU-IN2P3-INP (France); ASI, CNR, and INAF (Italy); NASA and DoE (USA); STFC and UKSA (UK); CSIC, MICINN, and JA (Spain); Tekes, AoF, and CSC (Finland); DLR and MPG (Germany); CSA (Canada); DTU Space (Denmark); SER/SSO
(Switzerland); RCN (Norway); SFI (Ireland); FCT/MCTES (Portugal); and PRACE (EU). A description of the Planck Collaboration and a list of its members, including the technical or scientific activities in which they have been involved, can be found at http://www.sciops.esa.int/index.php? project=planck\&page=Planck_Collaboration. W. Zhao and L. Santos are supported by project 973 under Grant No. 2012CB821804, by the NSFC No. 11173021, No. 11322324 and No. 11421303, and by a project of KIP of CAS. T. Villela acknowledges the support of CNPq through grant 308876/2014-8.

\section{References}

Abramo, L. R., Bernui, A., Ferreira, I. S., et al. 2006, Phys. Rev. D, 74, 063506 Akrami, Y., Fantaye, Y., Shafieloo, A., et al. 2006, ApJ, 784, L42

Aluri, P. K., \& Jain, P. 2012, MNRAS, 419, 3378

Axelsson, M., Ihle, H. T., Scodeller, S., \& Hansen, F. K. 2015, A\&A, 578 A44

Bennett, C. L., Halpern, M., Hinshaw, G., et al. 2003, ApJS, 148, 1

Bernui, A., Villela, T., Wuensche, C. A., et al. 2006, A\&A, 454, 409

Bernui, A., Oliveira, A. F., \& Pereira, T. S. 2014, J. Cosmol. Astropart. Phys., 10,41

Bielewicz, P., Górski, K. M., \& Banday, A. J. 2004, MNRAS, 355, 1283

Bielewicz, P., Eriksen, H. K., Banday, A. J., et al. 2005, ApJ, 635, 750

Copi, C. J., Huterer, D., \& Starkman, G. D. 2004, Phys. Rev. D, 70, 043515

Copi, C. J., Huterer, D., Schwarz, D. J., \& Starkman G. D. 2006, MNRAS, 367, 79

Cruz, M., Martínez-González, E., Vielva, P., \& Cayón, L. 2005, MNRAS, 356, 29

Cruz, M., Cayón, L., Martínez-González, E., et al. 2007, ApJ, 655, 11

de Oliveira-Costa, A., Tegmark, M., Zaldarriaga, M., \& Hamilton, A. 2004, Phys. Rev. D, 69, 063516

Donoghue, E. P., \& Donoghue, J. F. 2005, Phys. Rev. D, 71, 043002

Eriksen, H. K., Hansen, F. K., Banday, A. J., et al. 2004a, ApJ, 605, 14

Eriksen, H. K., Novikov, D. I., Lilje, P. B., et al. 2004b, ApJ, 612, 64

Frommert, M., \& Ensslin, T. A. 2010, MNRAS, 403, 1739

Gorski, K. M., Hivon, E., Banday, A. J., et al. 2005, ApJ, 622, 759

Gruppuso, A., \& Burigana, C. 2009, J. Cosmol. Astropart. Phys., 8, 4

Gruppuso, A., Finelli, F., Natoli, P., et al. 2011, MNRAS, 411, 1445

Gurzadyan, V. G., Kashin, A. L., Khachatryan, H. et al. 2014, A\&A, 566, A135

Hansen, F. K., Cabella, P., Marinucci, D., \& Vittorio, N. 2004a, ApJ, 607, L67

Hansen, F. K., Banday, A. J., \& Górski, K M. 2004b, MNRAS, 354, 641

Hansen, M., Kim, J., Frejsel, A. M., Ramazanov, S. et al. 2012, J. Cosmol. Astropart. Phys., 10, 049

Hinshaw, G., Nolta, M. R., Bennett, C. L., et al. 2007, ApJS, 170, 288

Hinshaw, G., Weiland, J. L., Hill, R. S., et al. 2009, ApJS, 180, 225

Hoftuft, J., Eriksen, H. K., Banday, A. J., et al. 2009, ApJ, 699, 9856

Jarosik, N., Bennett, C. L., Dunkley, J., et al. 2011, ApJS, 192, 14

Kim, J., \& Naselsky, P. 2010, ApJ, 714, L265

Land, K., \& Magueijo, J. 2005, MNRAS, 357, 994

Naselsky, P., Zhao, W., Kim, J., \& Chen, S. 2012, ApJ, 749, 31

Paci, F., Gruppuso, A., Finelli, F., et al. 2010, MNRAS, 407, 399

Pietrobon, D., Cabella, P., Balbi, A., et al. 2010, MNRAS, 402, 34

Planck Collaboration I. 2014, A\&A, 571, A1

Planck Collaboration XXIII. 2014, A\&A, 571, A23

Planck Collaboration IX. 2015, A\&A, submitted [arXiv: 1502.05956]

Planck Collaboration XIII. 2015, A\&A, submitted [arXiv: 1502 . 01589]

Planck Collaboration XVI. 2015, A\&A, in press,

DOI: $10.1051 / 0004-6361 / 201526681$

Polastri, L., Gruppuso, A., \& Natoli, P. 2015, JCAP, 4, 18

Quartin, M., \& Notari, A. 2015, J. Cosmol. Astropart. Phys., 1, 8

Rassat, A., Starck, J. -L., Paykari, P., et al. 2014, J. Cosmol. Astropart. Phys., 8, 6

Santos, L., Villela, T., \& Wuensche, C. A. 2012, A\&A, 544, A121

Santos, L., Cabella, P., Villela, T., et al. 2014, A\&A, 569, A75

Schwarz, D. J., Starkman, G. D., Huterer, D., \& Copi, C. J. 2004, Phys. Rev. Lett., 93, 221301

Smoot, G. F., Bennett, C. L., Kogut, A., et al. 1992, ApJ, 396, L1

Vielva, P. 2010, Adv. Astron., 2010, 592094

Vielva, P., \& Sanz, J. L. 2010, MNRAS, 404, 895

Vielva, P., Martínez-González, E., Barreiro, R. B., et al. 2004, ApJ, 609, 22

Zhao, W. 2014, Phys. Rev. D, 89, 023010 